\title{
Temperature compensation of ultrasonic velocity during the malolactic fermentation process
}

\author{
M A Amer ${ }^{1}$, D Novoa-Díaz², J A Chávez ${ }^{2}$, A Turó², M J García-Hernández² \\ and J Salazar ${ }^{2}$ \\ ${ }^{1}$ Escola Universitària Salesiana de Sarrià, Passeig Sant Joan Bosco 74, 08017 Barcelona, Spain \\ 2 Sensor Systems Group. Department of Electronic Engineering. Universitat Politècnica de Catalunya, \\ Jordi Girona 1-3, 08034 Barcelona, Spain \\ E-mail:maamer@euss.es
}

Received 14 July 2015, revised 8 September 2015

Accepted for publication 12 October 2015

Published

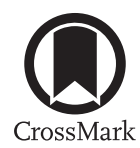

\begin{abstract}
Ultrasonic properties of materials present a strong dependence on temperature and in turn the ultrasonic velocity of propagation in the material under test. It is precisely for this reason that most ultrasonic measurements are often carried out with thermostated samples by using either water tanks or climate chambers. This approach is viable in a laboratory and when the measured or characterized samples are relatively small. However, this procedure is highly improbable to be applied when in situ measurements in industrial environments must be performed. This goes for the case of, for example, ultrasonic velocity measurements in wine while it is performing malolactic fermentation (MLF) inside a tank of hundreds of thousands of litres. In this paper two different practical approaches to temperature compensation are studied. Then, the two temperature compensation methods are applied to the measured ultrasonic velocity values along a whole malolactic fermentation process. The results of each method are discussed.
\end{abstract}

Keywords: malolactic fermentation, ultrasound, process monitoring, temperature compensation

(Some figures may appear in colour only in the online journal)

\section{Introduction}

Malolactic fermentation (MLF) is a biological process that consists of the transformation of malic acid into both lactic acid and carbon dioxide. This process, caused by lactic acid bacteria (LAB), takes place during the production of the majority of red wines as well as certain types of white wines, and contributes to the development of the sensory characteristics of wine (Wibowo et al 1985, Maicas et al 1999, Liu 2002, Lerm et al 2010, Vila-Crespo et al 2010).

In order to obtain a correct, controlled malolactic fermentation process, it is important to properly monitor it and detect when the reaction is finished. The task of monitoring the progress of MLF is mostly carried out by measuring the concentration of malic and lactic acids in wine samples and, for this purpose, several 'off-line' measurement methods are described in the literature (Lonvaud-Funel 2004, Lerm et al 2010).

Ultrasound has been recently proven as a successful technology for on-line MLF process monitoring (García-Álvarez et al 2011, Novoa-Díaz et al 2012, 2014a). In addition, the relationship between the ultrasonic velocity of propagation and malic and lactic acid concentration was previously studied by the authors of this paper (Amer et al 2015). As a result, an empirical equation was obtained and MLF process monitoring should be possible by measuring the ultrasonic velocity. Unfortunately, it is well known that ultrasonic velocity is also highly sensitive to temperature (Gross and Mader 1972, McClements 1997, Salazar et al 2009), and consequently, samples should be thermostated during the process.

\begin{tabular}{|lllll}
\hline JNL:MST & PIPS: AA0631 TYPE: PAP & TS: NEWGEN & DATE:24/10/15 & EDITOR: JW \\
\hline
\end{tabular}


This is a serious handicap and reduces their use in a laboratory environment.

In order to monitor MLF by using ultrasound technology in real fermentation processes, it is necessary to compensate the temperature effect of the ultrasonic velocity of propagation (Novoa-Díaz et al 2014a). Keeping the temperature controlled and constant (as in lab measurements) is not possible in real wine MLF processes. So, it is necessary to measure the temperature of the wine sample and then compensate mathematically the temperature contribution of the ultrasonic velocity of propagation. For this reason, the existing correlation between ultrasonic velocity variation and temperature variation in wine samples should be studied, and a temperature coefficient of compensation should be determined for each wine sample (Novoa-Díaz et al 2014a).

Two methods to obtain the temperature coefficient of compensation are described in this paper. The first one consists of measuring the ultrasonic velocity in synthetic hydroalcoholic solutions that are thermostated at different temperatures. Then, an empirical equation that correlated the temperature compensation coefficient and the alcoholic degree is obtained. So, from this equation it should be possible to estimate the temperature compensation coefficient if the alcoholic degree of the wine is known.

The second method is based in measuring simultaneously the ultrasonic velocity and the temperature of the wine sample during the MLF process, taking into account that variations due to temperature will be greater than those produced by the MLF itself. Then, it is possible to adjust 'on-line' and empirically a linear model that correlates both variables, where the slope is the temperature coefficient.

Finally, both methods of temperature compensation have been tested in two cases: The first one corresponds to MLF carried out in a pilot plant. The second one corresponds to the MLF of a real wine process. The results obtained in both samples are described, in order to evaluate the possibility of using the ultrasonic velocity of propagation for determining the end of point of the fermentation.

\section{Theoretical}

\subsection{Malolactic fermentation (MLF)}

2.1.1. Stoichiometry. As shown in equation (1), during MLF malic acid $\left(\mathrm{C}_{4} \mathrm{H}_{6} \mathrm{O}_{5}\right)$ is transformed into lactic acid $\left(\mathrm{C}_{3} \mathrm{H}_{6} \mathrm{O}_{3}\right)$ and carbon dioxide $\left(\mathrm{CO}_{2}\right)$. This process is catalysed by a highly specialized enzyme (the 'malolactic enzyme') and carried out by the LAB, mainly those of the Oenococcus, Lactobacillus, Pediococcus, and Leuconostoc strains (Wibowo et al 1985).

$$
\mathrm{C}_{4} \mathrm{H}_{6} \mathrm{O}_{5} \rightarrow \mathrm{C}_{3} \mathrm{H}_{6} \mathrm{O}_{3}+\mathrm{CO}_{2} .
$$

Stoichiometrically, 1 mole of malic acid produces 1 mole of lactic acid and 1 mole of carbon dioxide. But if mass concentrations are considered, a $3 \mathrm{~g}^{-1}$ reduction of malic acid equals to an increase of about $2 \mathrm{~g} \mathrm{l}^{-1}$ of lactic acid.
2.1.2. MLF process. Three steps are defined in MLF, correlative in time: (i) the bacterial growth phase, (ii) stationary phase I, and (iii) stationary phase II (Krieger 2006).

(i) Bacterial growth phase:

This phase starts when lactic acid bacteria (LAB) are inoculated. LAB growth takes place during this phase. This results in a consumption of sugars that were not fermented during the alcoholic fermentation phase. A small amount of acetic acid is also produced. No malic acid is metabolized, so the malic acid and lactic acid concentrations are stable.

(ii) Stationary phase I:

This phase starts when the bacterial growth phase is finalized. During this phase, the amount of LAB is stable and malic acid is transformed into lactic acid. No sugar consumption is produced (LAB prefer malic acid).

(iii) Stationary phase II:

This is the last phase. During this third phase, no more malic acid is transformed into lactic acid, but citric acid is degraded and acetic acid is produced. Also, the amount of LAB is reduced. This phase should be avoided in wineries, because the wine characteristics are degraded.

So, it is important to determine the end point of phase (ii), in order to prevent phase (iii) from happening.

2.1.3. Control of MLF. The decarboxylation of the malic acid in wine is the most obvious action of MLF. The easiest way to monitor the progress of the MLF is to chemically analyze the disappearance of malic acid and the formation of lactic acid. The most commonly used quantitative analytical method for monitoring MLF is the enzymatic determination of L-malic acid (OIV 2012). This method uses an enzyme that specifically reacts with L-malic acid and a UV-visible spectrophotometer to monitor the progress of the analytical reaction. The detection of L-malic acid requires two enzyme reactions. In the first reaction, malic acid (L-malate) is oxidized to oxaloacetate by nicotinamide-adenine dinucleotide (NAD) in the presence of L-malate dehydrogenase (L-MDH):

L-Malate $+\mathrm{NAD}^{+} \stackrel{\text { L-MDH }}{\longleftrightarrow}$ oxaloacetate $+\mathrm{NADH}+\mathrm{H}^{+}$.

However, since the equilibrium of reaction (equation (2)) lies firmly in favour of L-malate and $\mathrm{NAD}^{+}$, a further reaction is required to trap the NADH product, and this is achieved by the conversion of oxaloacetate to L-aspartate and 2-oxoglutarate, in the presence of a large excess of L-glutamate, by glutamateoxaloacetate transaminase (GOT):

$$
\begin{aligned}
& \text { Oxaloacetate }+ \text { L-glutamate } \stackrel{\mathrm{GOT}}{\longleftrightarrow} \text { L-aspartate } \\
& \quad+\text { 2-oxoglutarate. }
\end{aligned}
$$

The amount of NADH formed is stoichiometric to the amount of L-malate. The increase in NADH is measured through the measurement of its light absorbance at 334,340 , or $365 \mathrm{~nm}$. 


\subsection{Ultrasonic velocity in liquid media}

When the distance travelled by an ultrasonic wave through a liquid medium is a known constant, the wave's velocity can be calculated using equation (4)

$$
v=\frac{d_{\text {travelled }}}{\text { TOF }},
$$

where TOF corresponds to the time of flight, which is the time taken by a wave to travel a given distance $\left(d_{\text {travelled }}\right)$. A series of practical methods to measure TOF were described and analyzed in a previous paper (Novoa-Díaz et al 2012), as was the method for determining ultrasonic velocity.

Generally, TOF varies in accordance with the physical and chemical changes in the medium. Given this, variations of lactic and malic acid concentrations in the liquid mixture will cause changes to the TOF, and consequently, to the ultrasonic wave velocity. Generally speaking, the propagation parameters for ultrasonic waves in the medium are a composite of the separate contributions made by each individual element present in the medium (Resa et al 2007). In addition, temperature changes affect the acoustic properties of materials, and consequently their ultrasonic velocity.

\section{Materials and methods}

\subsection{Preliminary}

This section is divided into three parts. The first one (section 3.2) describes the materials and methods performed in synthetic laboratory hydroalcoholic samples. The second section 3.3 describes the materials and methods performed using real wine MLF samples. Finally, in section 3.4, the method used for measuring the ultrasonic velocity of propagation is described.

\subsection{Laboratory measurements}

3.2.1. Samples. The reagents used to prepare the mixtures were distilled water and Ethanol 96\% v/v PA-ACS (Panreac Química S.L.U., Barcelona, Catalonia, Spain). Ethanol solutions at $5 \%, 7 \%, 10 \%$, and $15 \%$ v/v were prepared from Ethanol $96 \%$ using $1000 \mathrm{ml}$ and $100 \mathrm{ml}$ volumetric flasks, and 5 and $10 \mathrm{ml}$ pipettes (Duran, Germany).

3.2.2. Experimental cell. Ethanol samples at 5\%, 7\%, 10\%, and $15 \% \mathrm{v} / \mathrm{v}$ were placed in a $600 \mathrm{ml}$ glass beaker, which was immersed in a thermostatic bath (Omega, Stamford, UK) The temperature of the bath was increased from $20^{\circ} \mathrm{C}$ up to $32{ }^{\circ} \mathrm{C}$. An ultrasonic transducer (B1F, General Electric, USA) was connected to a buffer rod and placed in contact with the hydroalcoholic samples. The transducer is excited at its resonant frequency with a sine wave tone burst and the resulting echo is measured (see section 3.4). The temperature of the solutions is measured using a Fluke 1551 A Pt100 Thermometer, with a $0.05{ }^{\circ} \mathrm{C}$ precision.

\subsection{Real wine MLF processes measurements}

3.3.1. Wine samples. Two wine samples are considered. The first one is a Tempranillo wine (Palencia, Castilla, Spain). The second one is a Syrah wine (Penedès, Catalunya, Spain). Alcoholic fermentation is concluded in both samples.

3.3.2. Experimental cell. The wine samples indicated in section 3.3.1 were placed in industrial tanks of various capacities. In order to measure the ultrasonic wave propagation, the sensor should be placed inside the tank. It is for this reason that a custom head was designed and introduced into the tank using one of the existing 50 DIN butterfly valves. The ultrasonic sensor (based on a B1F ultrasonic transducer, General Electric, USA) was placed inside the head, and connected with a buffer rod in contact with the wine samples. The ultrasonic sensor was excited at its resonant frequency with a sine-wave tone burst and the resulting echo was measured (see section 3.4).

In order to measure the wine temperature, the head was also equipped with a $5.0 \mathrm{~K} \Omega$ thermistor, placed in contact with the wine sample. The electrical signal from the thermistor was measured using a data-logger (Agilent 34970A/34972A Data Acquisition).

During the MLF process, the malic and lactic acid concentrations were chemically determined from the enzymatic method described in section 2.1.3. Kits from manufacturers that contain all the reagents, enzymes, and procedures required for L-malic acid determinations are readily available. For this study, two separate kits were used (one for each reagent): An L-Malic Acid Enzymatic Kit (Boehringer Mannheim-Roche, Spain) and an L-Lactic Acid Enzymatic Kit (Boehringer Mannheim-Roche, Spain). In addition, a multiparametric analyzer Lisa 200 (Hycel diagnostics, TDI Tecnología Difusión Ibérica, S.L., Spain) was used.

3.3.3. Data acquisition. The instruments described in section 3.3.2 were connected to a PC using USB buses. Data acquisition was performed by a custom application developed using the LabVIEW environment from National Instruments. The program interface is shown in figure 1. Figure 2 represents schematically the program structure that was developed

According to figure 2, first the program opens communication with the connected instruments, such as the oscilloscope, data-logger, and function generator. Also, a data file path is created.

The second step consists of the instruments' configuration. A default configuration is established for each instrument automatically, but the user can modify it as many times as desired before the data acquisition began. The sampling rate, scope channels selected, and number of samples read in each acquisition are established at this step.

Data acquisition begins manually when the 'acquire' button is pressed by the user. Then, instrument configuration changes are no longer possible. During the data acquisition period, data are automatically captured at the sampling rate previously established. Two data types are acquired at each 


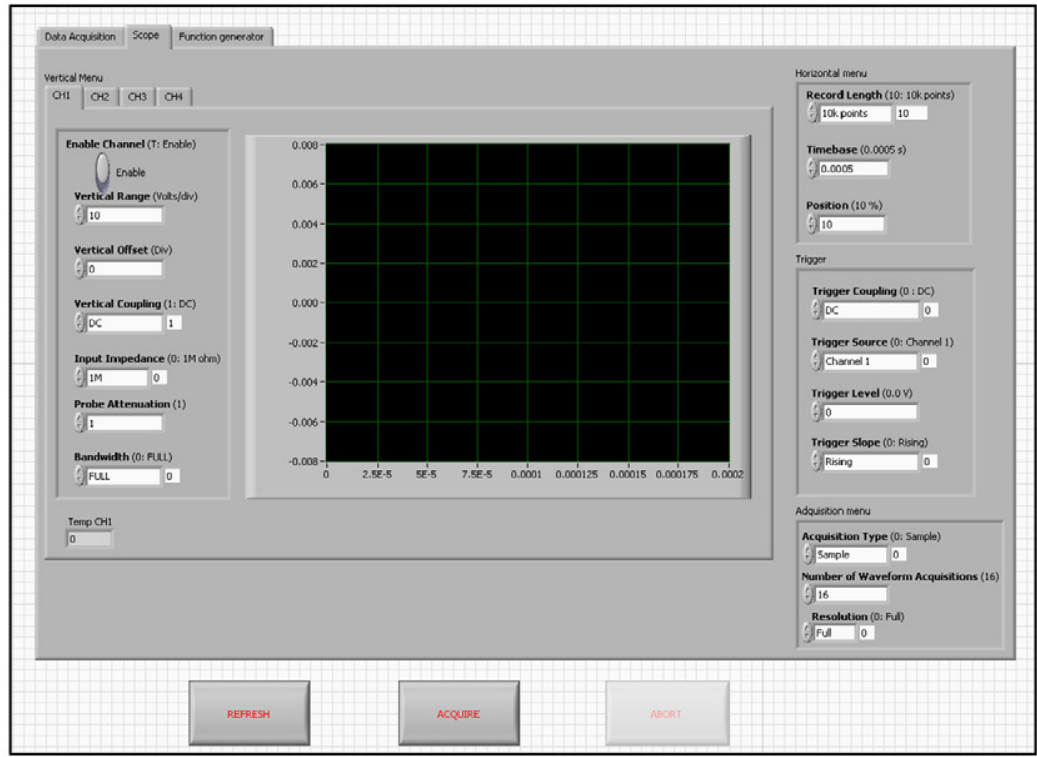

Figure 1. Capture of the program interface developed for acquiring signals from instruments.

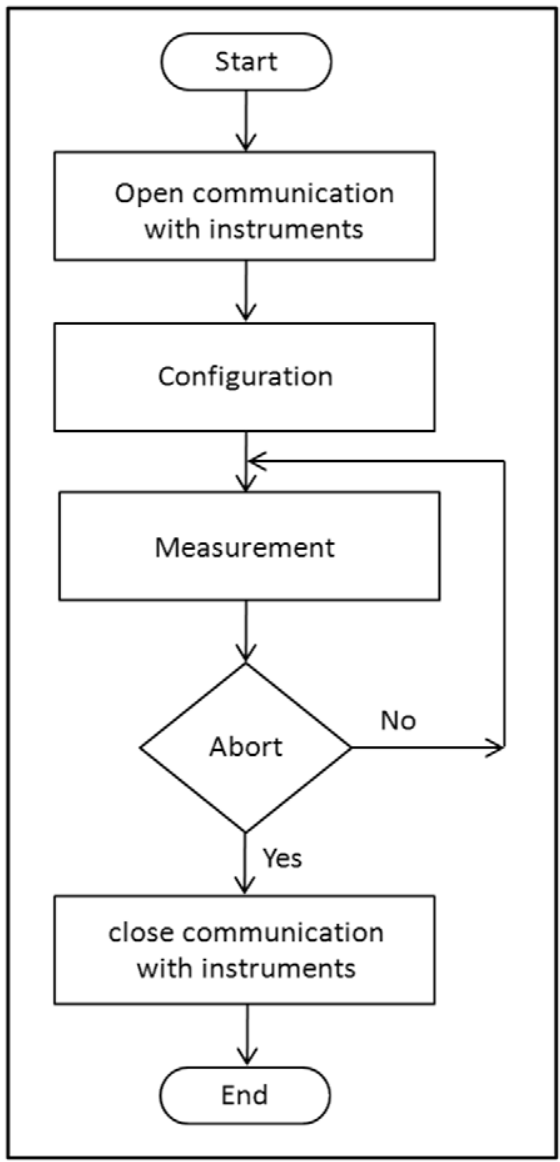

Figure 2. Program structure.

sampling time: Wine temperature (provided by the datalogger) and the ultrasonic waveform (provided by the scope).

The acquired data are saved in spreadsheet compatible files, one for each capture. All the files are saved in the same path, have the same root name, and are distinguished by a number correlated in time. The data file format equals to a spreadsheet one, where each column corresponds to a scope channel.

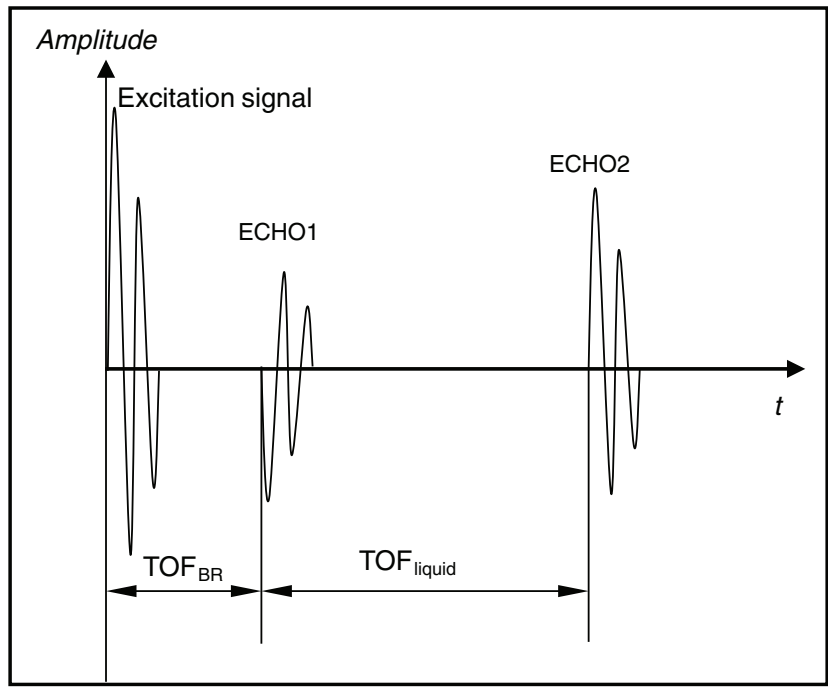

Figure 3. Ultrasonic velocity measurement. $\mathrm{TOF}_{\mathrm{BR}}$ : time-of-flight in the buffer rod. $\mathrm{TOF}_{\text {liquid: }}$ time-of-flight in the liquid solution.

For each channel, it is saved: The channel name, the data and time when the acquisition took place, the sample temperature, the interval rate between correlative points, and the series of the scope data points are displayed.

The data acquisition process runs indefinitely until it is aborted manually by the user. Then, the communication with the instruments is closed and program execution is finished.

\subsection{Ultrasonic velocity measurement}

The method for the determination of the ultrasonic velocity was based on a pulse-echo technique using a tone-burst pulse. The head sensor contains an emitter-receiver ultrasound transducer attached to a cylindrical buffer rod. The transducer was excited at its $1 \mathrm{MHz}$ resonant frequency with a sine-wave tone burst of 10 cycles and $20 \mathrm{Vpp}$ of amplitude, using an Agilent 33522 function/Arbitrary Waveform Generator. 


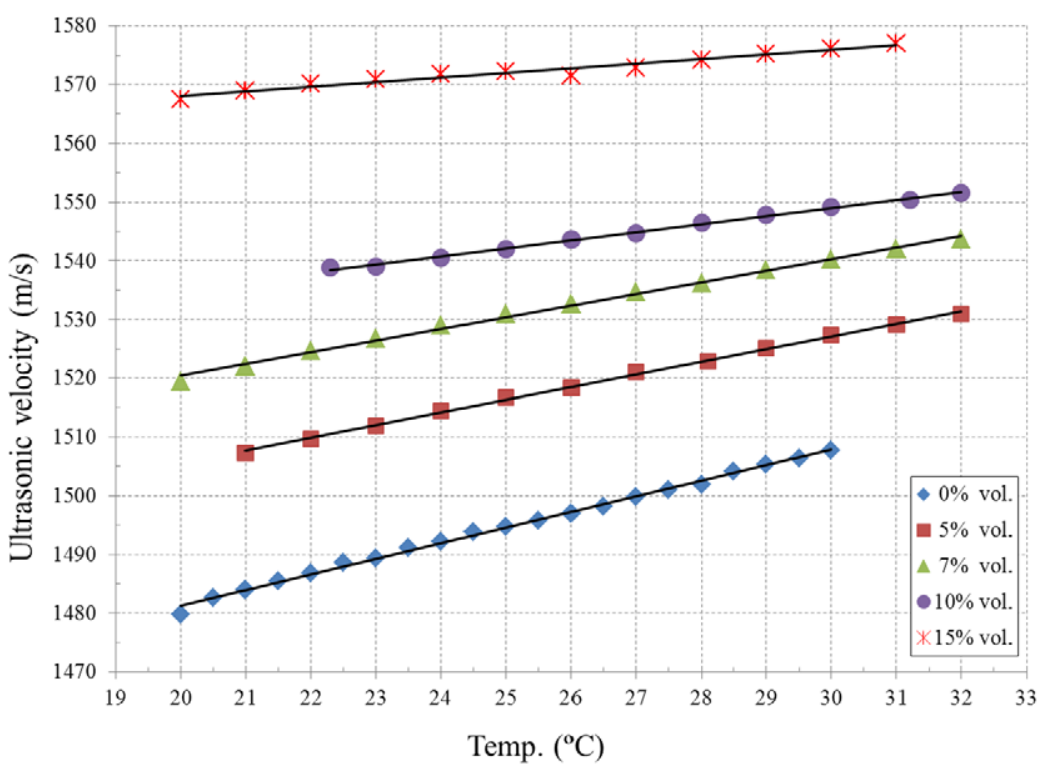

Figure 4. Linear correlations ultrasonic velocity/temperature for hydroalcoholic samples at different alcoholic degrees.

The generated acoustic wave propagates along the buffer rod until it reaches the buffer rod-liquid interface. Then, part of the incident wave is reflected back to the ultrasonic transducer (ECHO1) and the other part is transmitted through the liquid sample until it reaches the surface of an acoustic reflector. At the reflector, the transmitted wave is reflected back towards the liquid-buffer rod interface, where once again part of this signal is transmitted through the buffer rod and detected by the ultrasound transducer $(\mathrm{ECHO} 2)$. The received waves were acquired using a Tektronix $200 \mathrm{MHz} / 1 \mathrm{Gs} \mathrm{s}^{-1}$ DPO 2024 Digital Phosphor Oscilloscope (figure 3).

Finally, the acquired signals were analyzed using a fast Fourier transform (FFT) algorithm to obtain the time of flight in the liquid ( $\mathrm{TOF}_{\text {liquid }}$ ), as described in previous work (Novoa-Díaz et al 2012). Then, the ultrasonic propagation velocity of a wave in the liquid was calculated by dividing the travelled distance through the liquid by the time of flight, as stated in equation (4). The ultrasonic propagation velocity was calculated with an uncertainty of less than $\pm 0.1 \mathrm{~m} \mathrm{~s}^{-1}$ (Novoa-Díaz 2014b).

\section{Results and discussion}

\subsection{Preliminary}

This section is divided into two parts. The first one describes the two methods used to obtain the temperature compensation coefficients: From synthetic hydroalcoholic solutions and from correlations between ultrasonic velocity and temperature in real wine samples. The second part studies the use of the temperature compensation coefficients in real wine samples in order to monitor the MLF process.

\subsection{Determination of temperature compensation coefficients}

4.2.1. From synthetic hydroalcoholic samples. The purpose of using synthetic hydroalcoholic solutions was mainly to
Table 1. Adjusted empirical functions and correlation coefficients $\mathrm{R}^{2}$, for ultrasonic propagation of velocity ( $y$-axis) related to temperature ( $x$-axis), for hydroalcoholic samples at different alcoholic degrees. Unities: $x\left({ }^{\circ} \mathrm{C}\right), y\left(\mathrm{~m} \mathrm{~s}^{-1}\right)$.

\begin{tabular}{lll}
\hline & Empirical function & $R^{2}$ \\
\hline Ethanol 0\% v/v & $y=2.6586 x+1428.1$ & 0.9967 \\
Ethanol $5 \% \mathrm{v} / \mathrm{v}$ & $y=2.1623 x+1462.3$ & 0.9986 \\
Ethanol 7\% v/v & $y=1.9866 x+1480.7$ & 0.9960 \\
Ethanol 10\% v/v & $y=1.3700 x+1507.9$ & 0.9966 \\
Ethanol 15\% v/v & $y=0.7806 x+1552.5$ & 0.9625 \\
\hline
\end{tabular}

obtain a general trend about a theoretical ultrasonic velocity, which could be used later in the second part of this study to validate the temperature compensation strategy. So, in order to estimate the relationship between the ultrasonic velocity of propagation and the temperature, aqueous ethanol samples in different concentrations $(0 \%, 5 \%, 7 \%, 10 \%$, and $15 \% \mathrm{v} / \mathrm{v})$ are prepared (see section 3.2). These samples are thermostated at different temperatures, and the ultrasonic velocity of propagation is measured. The results are represented graphically in figure 4, and a good linear correlation is observed.

The empirical equations from the data obtained in figure 4 are derived using a linear model. The derived equations and the correlation coefficients $R^{2}$ are shown in table 1. From those empirical equations, the slope corresponds to the temperature compensation coefficient for each sample. That is, the slope determines the contribution of the temperature factor to the ultrasonic velocity variation.

Table 1 shows the temperature coefficients of compensation for synthetic hydroalcoholic samples at specific alcoholic degrees $(0 \% \mathrm{v} / \mathrm{v}, 5 \% \mathrm{v} / \mathrm{v}, 7 \% \mathrm{v} / \mathrm{v}, 10 \% \mathrm{v} / \mathrm{v}$, and $15 \% \mathrm{v} / \mathrm{v})$. For other alcoholic degrees, the whole experimental procedure should be repeated. Alternatively, it is possible to obtain empirically the relationship between the temperature compensation coefficient and the alcoholic degree. So, the temperature compensation coefficients obtained from table 1 are represented graphically (figure 5). 


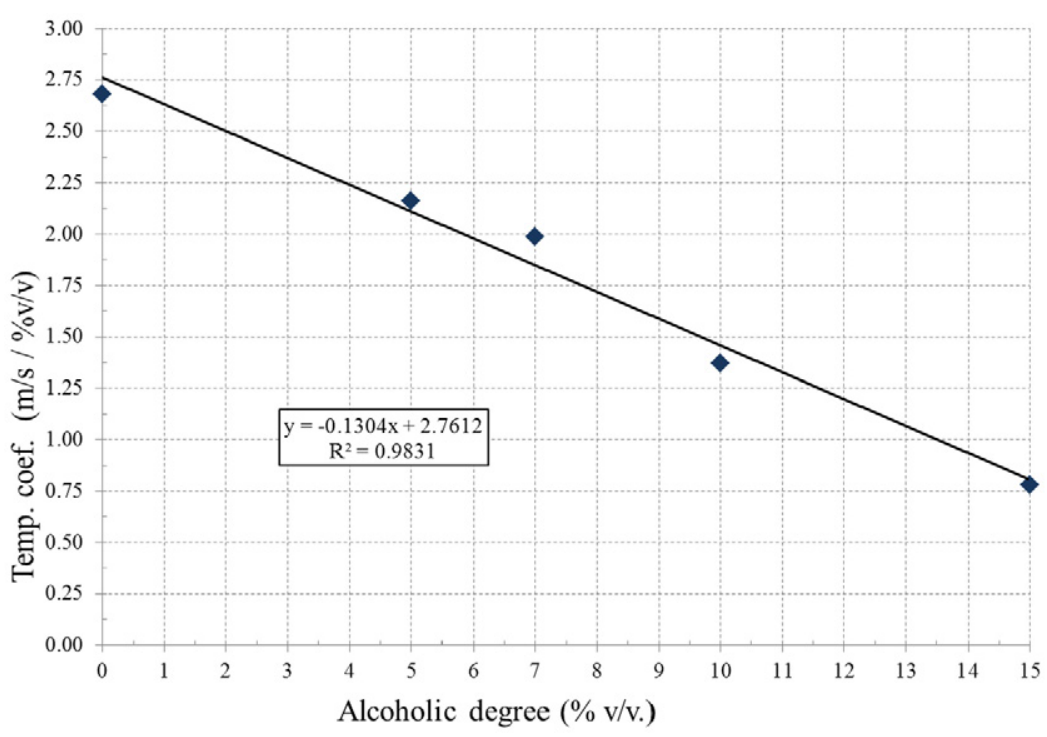

Figure 5. Representation of the temperature coefficients of compensation at different alcoholic degrees.

From the data represented in figure 5, a linear empirical equation has been derived (equation (5)), with a good correlation coefficient $\left(\mathrm{R}^{2}\right.$ is higher than 0.98$)$.

$$
m=-0.1304 \cdot d+2.7612 .
$$

In equation (5), $\mathrm{m}$ refers to the temperature compensation coefficient (in $\mathrm{m} \mathrm{s}^{-1}{ }^{\circ} \mathrm{C}^{-1}$ ) and d refers to the alcoholic degree (in $\% \mathrm{v} / \mathrm{v}$ ). So, it is possible to obtain the temperature coefficient of compensation from equation (5) if the alcoholic degree is known and, consequently, to eliminate the temperature effect from the ultrasonic velocity of propagation.

From the temperature coefficient of compensation obtained in equation (5), it is possible to compensate the temperature effect in the ultrasonic velocity of propagation, as described by equation (6).

$$
v_{i \mathrm{c}}=v_{i}-\left(t_{i}-t_{\mathrm{o}}\right) \cdot m .
$$

In equation (6), $v_{i c}$ refers to the ultrasonic velocity of sample $i$ after the temperature compensation is applied, $v_{i}$ refers to the ultrasonic velocity of sample $i$ before the temperature compensation is applied, $t_{i}$ refers to the temperature of sample $i, t_{\mathrm{o}}$ refers to the temperature at the beginning of the experiment, and $m$ is the temperature coefficient of compensation obtained from equation (5).

\subsubsection{From correlations between ultrasonic velocity and} temperature in real wine samples. The method described in section 4.2.1 obtains the temperature compensation coefficients from an empirical equation (equation (5)), which is derived from the synthetic hydroalcoholic samples. So, the values obtained from this equation should not be valid for real wine samples.

So, in order to obtain valid temperature coefficients of compensation, the ultrasonic velocity of propagation should be measured at different temperatures using a real wine sample and then, from the results obtained, a linear equation should be adjusted. The slope of this equation is the temperature coefficient for the specific wine sample that is derived.
The disadvantage of the method described above is that the whole experimental process of obtaining the temperature coefficient of compensation should be repeated for each new wine sample. So, for a real wine MLF process, the temperature coefficient of compensation should be obtained off-line before starting fermentation. This is counter to the process of automation, since the winemaker would have to obtain the temperature coefficient manually and prior to monitoring the wine fermentation.

Alternatively, another way to obtain the temperature coefficient of compensation experimentally from real wine samples is presented. The method is very simple, and it is based on measuring simultaneously the ultrasonic velocity of propagation and the temperature during the MLF process. Then, the temperature and the ultrasonic velocity results obtained should be correlated in order to adjust an empirical equation. The slope of the equation is the temperature coefficient of compensation.

This method allows the evaluation of an on-line temperature coefficient of compensation automatically as the fermentation proceeds. For each new value of velocity and temperature obtained, a new empirical equation should be adjusted, and a new temperature coefficient should be recalculated.

This method was tested in two real wine samples where MLF took place. The results obtained are described below.

The first sample corresponds to a 'Tempranillo' wine from Palencia (see section 3.3). In this sample, the ultrasonic velocity of propagation and the temperature were measured during the MLF process (figure 6).

From figure 6, it is observed that the ultrasonic velocity and temperature profiles are similar. This is because the ultrasonic velocity is highly temperature dependent. Accordingly, during the MLF process, the temperature has changed significantly (with a maximum increase of $4{ }^{\circ} \mathrm{C}$ ), and the ultrasonic velocity variation is mainly due to the temperature variation.

From results collected in figure 6 , it is possible to correlate ultrasonic velocity and temperature. Figure 7 shows the obtained correlation. 


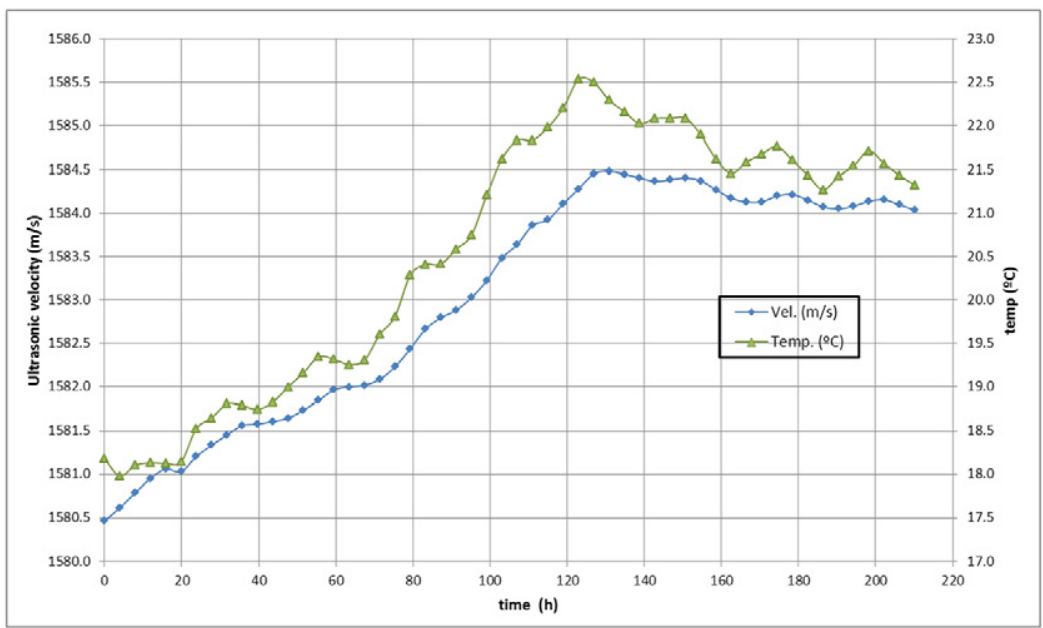

Figure 6. Measured ultrasonic velocity and temperature during the MLF process (Palencia wine sample).

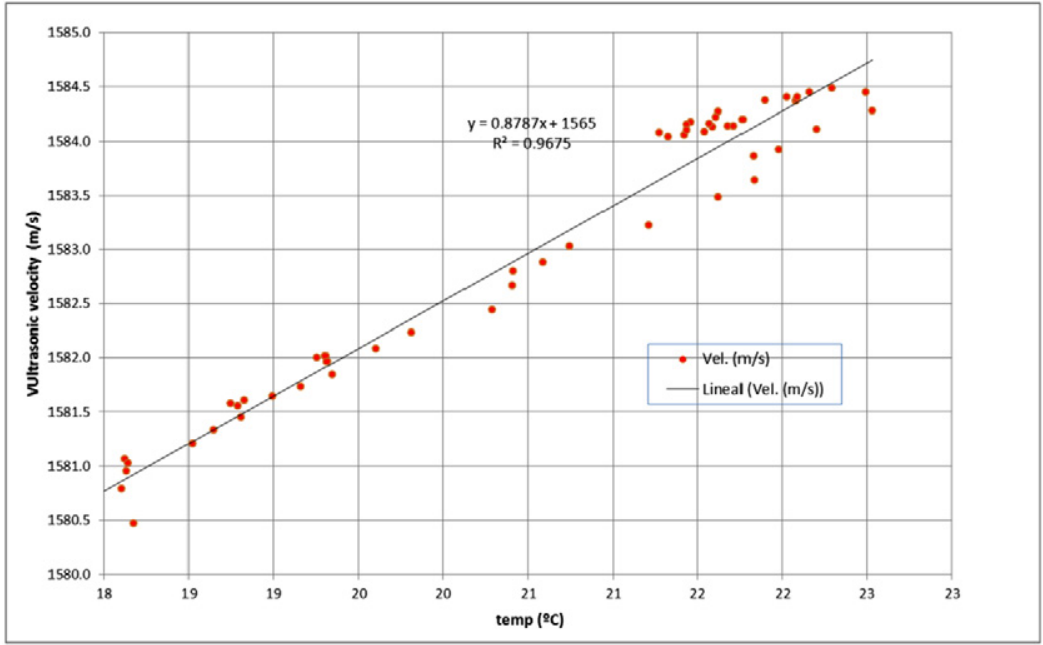

Figure 7. Correlation obtained between ultrasonic velocity of propagation and temperature (Palencia wine sample).

In figure 7, a good correlation between ultrasonic velocity and temperature is observed. This is consistent with the above comment, because temperature is the main factor that affects the ultrasonic velocity. So, a linear empirical equation is derived (equation (7)).

$$
v=0.8787 \cdot t+1565.0 \text {. }
$$

In equation (7), $v$ refers to the ultrasonic velocity of propagation (in $\mathrm{m} \mathrm{s}^{-1}$ ) and $t$ refers to the temperature (in ${ }^{\circ} \mathrm{C}$ ). The correlation coefficient $R^{2}$ is 0.9675 . So, the slope of the linear equation indicates that the ultrasonic velocity increases with a rate of $0.88 \mathrm{~m} \mathrm{~s}^{-1}$ per one degree Celsius of temperature. This is the temperature coefficient of compensation for the Palencia wine sample, experimentally obtained from the collected MLF data. This coefficient can be used to compensate the temperature effect in the ultrasonic velocity of propagation by applying equation (6).

The alcoholic degree of the Palencia wine sample is also known, and its value is $14.18^{\circ} \mathrm{v} / \mathrm{v}$. So, applying equation (5) it is possible to estimate a temperature coefficient of compensation; and the value obtained is $0.91 \mathrm{~m} \mathrm{~s}^{-1}$ per one degree Celsius. This value is rather similar to the experimental value obtained from equation (6) ( $0.88 \mathrm{~m} \mathrm{~s}^{-1}$ per one degree Celsius), and so both methods to obtain the temperature coefficient give similar results for this wine sample.

The second wine sample tested corresponds to a 'Syrah' wine from Penedès (see section 3.3). In contrast with the Palencia wine, in this case the MLF took place in an open-air winery, and so it was subjected to significant temperature variations between day and night. Figure 8 shows the ultrasonic velocity of propagation as a function of time, and figure 9 shows the temperature variation, also with respect to time.

The data collected in figure 8 shows that the ultrasonic velocity oscillated with time over a $24 \mathrm{~h}$ period, similar to the temperature variation (figure 9). This is consistent with the thermal variations between day and night, because the MLF took place in an open-air winery (as previously described), and temperature is the main factor that affects the ultrasonic velocity of propagation. In this case, the temperature fluctuations are much more pronounced than in the previous sample of Palencia and this leads to more difficult data processing.

If both figures were overlapped, some similar features are observed. This is quite obvious, because ultrasonic velocity is highly temperature dependent. 


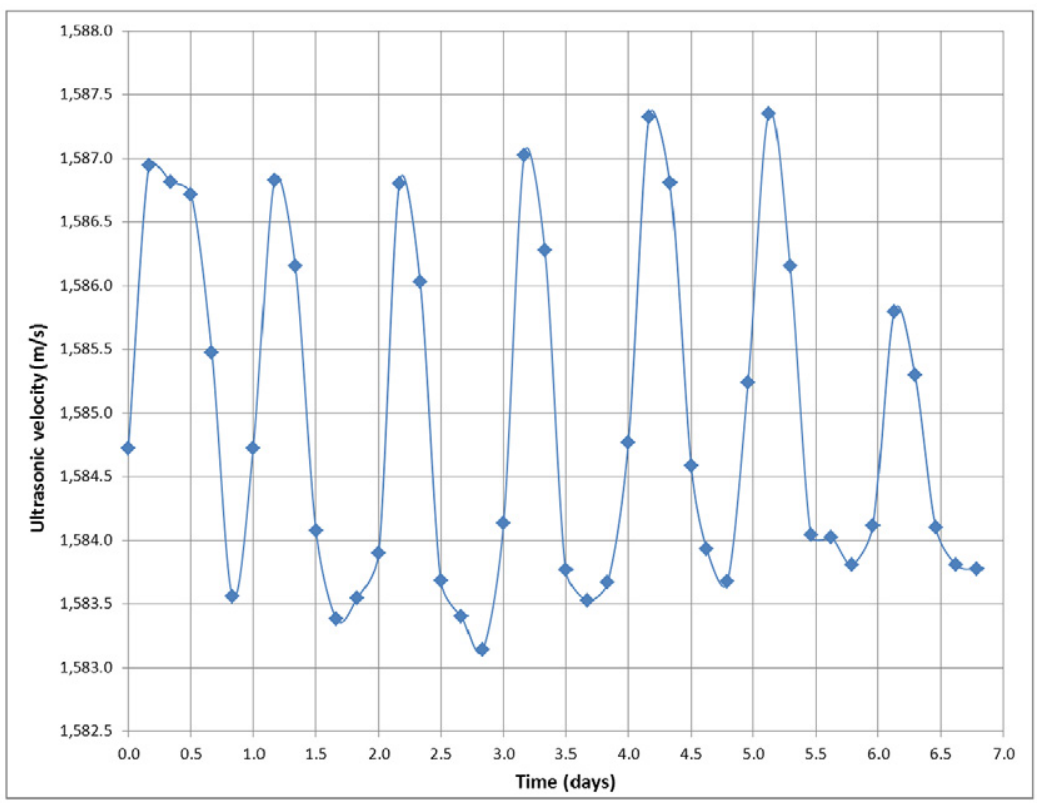

Figure 8. Ultrasonic velocity as a function of time along the MLF (Penedès wine sample).

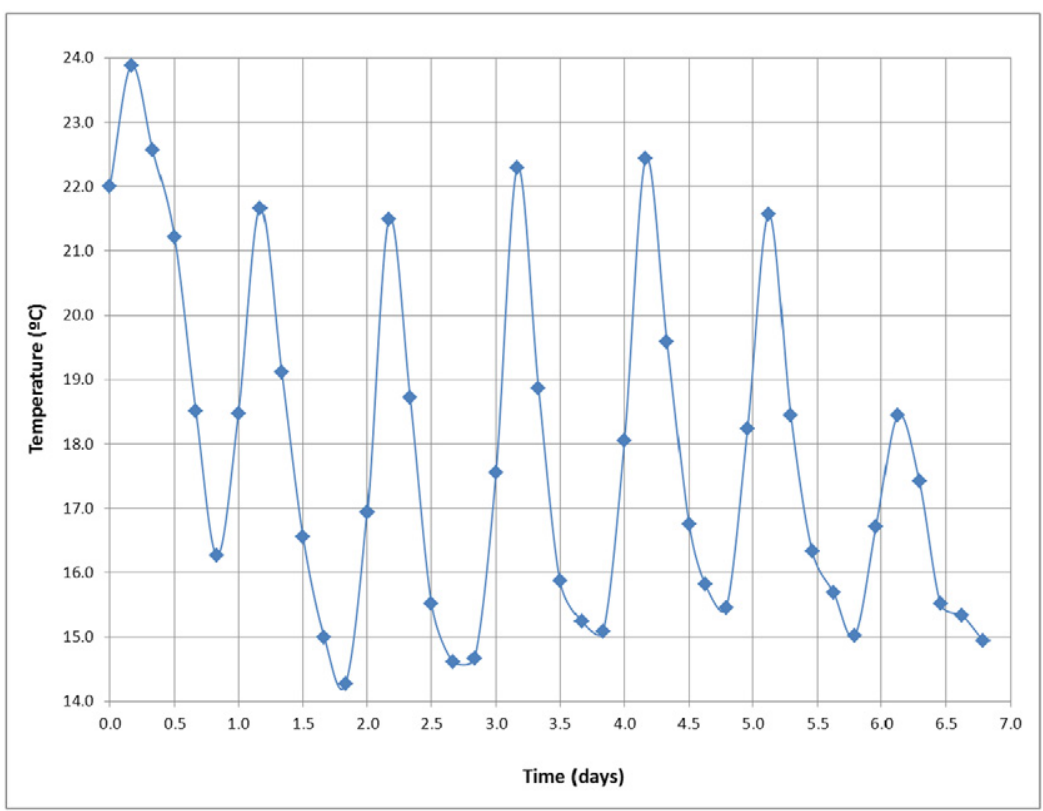

Figure 9. Temperature variation as a function of time (Penedès wine sample).

Similar to the Palencia wine sample, figure 10 shows the results obtained when ultrasonic velocity is correlated to temperature.

From results obtained in figure 10, an empirical linear equation is derived (equation (8)).

$$
v=0.519 \cdot t+1578.8 \text {. }
$$

In equation (8), $v$ refers to the ultrasonic velocity of propagation (in $\mathrm{m} \mathrm{s}^{-1}$ ) and $t$ refers to the temperature (in ${ }^{\circ} \mathrm{C}$ ). The correlation coefficient $R^{2}$ is 0.9093 . So, the slope of the linear equation indicates that the ultrasonic velocity increases with a rate of $0.52 \mathrm{~m} \mathrm{~s}^{-1}$ per one degree Celsius of temperature. This is the temperature coefficient of compensation for the
Penedès wine sample, experimentally obtained from the collected MLF data. This coefficient can be used to compensate the temperature effect in the ultrasonic velocity of propagation by applying equation (6).

The alcoholic degree of the Penedès wine sample is also known, and its value is $13.8^{\circ} \mathrm{v} / \mathrm{v}$. So, applying equation (5) it is possible to estimate a temperature coefficient of compensation, and the value obtained is $0.96 \mathrm{~m} \mathrm{~s}^{-1}$ per one degree Celsius. This value is almost double the experimental value obtained from equation (8) $\left(0.52 \mathrm{~m} \mathrm{~s}^{-1}\right.$ per one degree Celsius). Therefore, for this wine sample, other factors apart from the alcoholic degree are involved in the temperature coefficient estimation. 


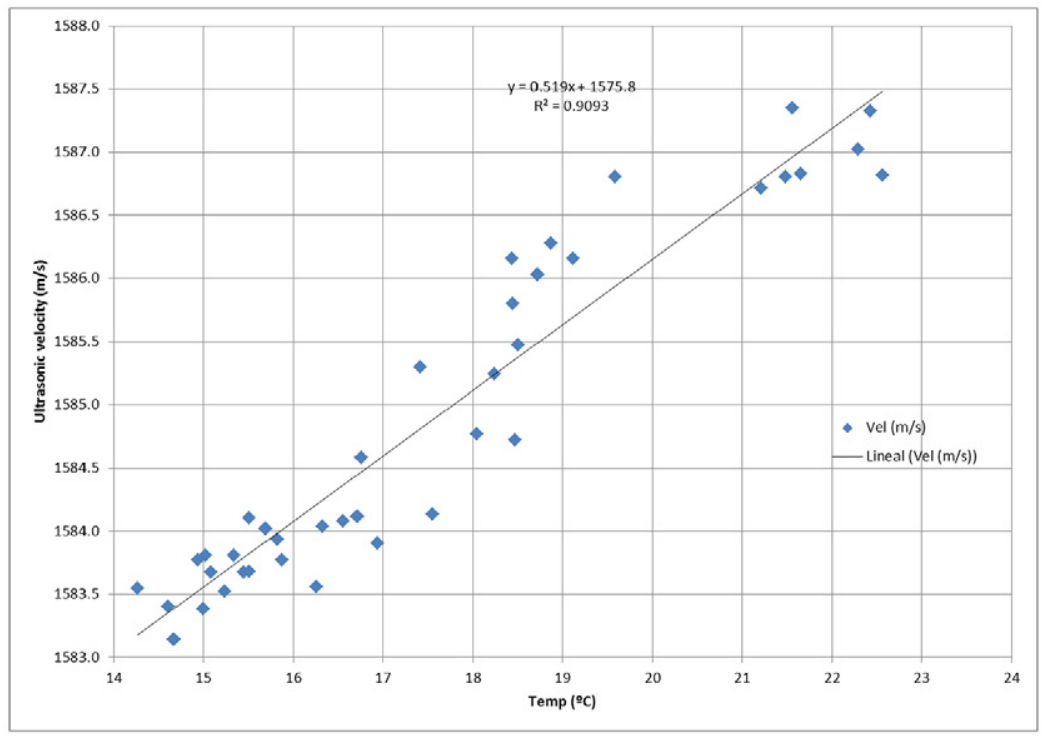

Figure 10. Correlation between ultrasonic velocity and temperature (Penedès wine sample).

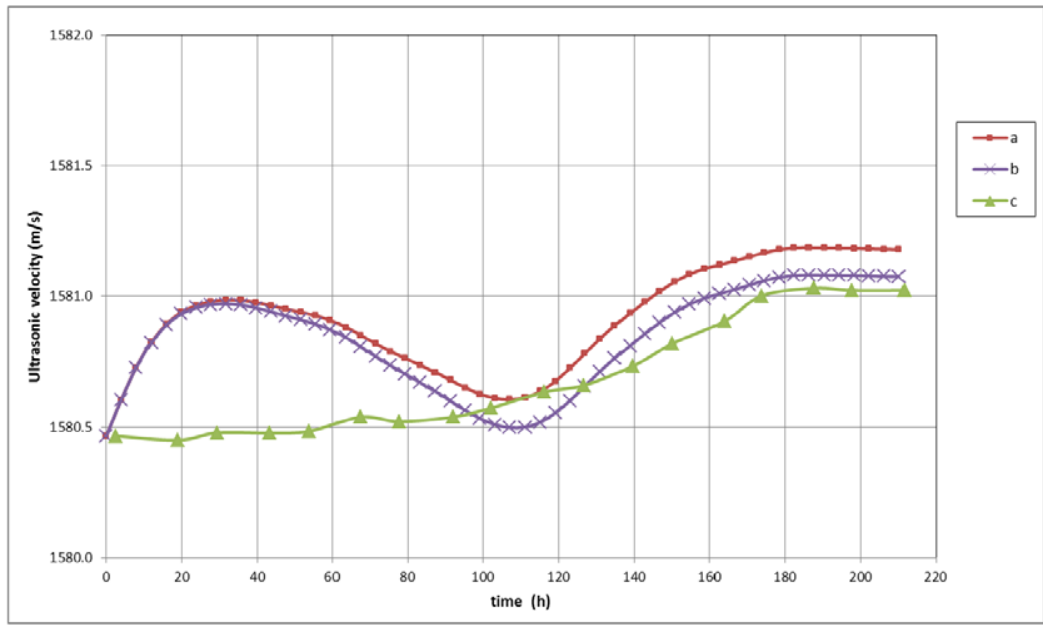

Figure 11. Ultrasonic velocity of propagation in the Palencia wine sample. (a) Temperature compensated data, applying a temperature coefficient of $0.88 \mathrm{~m} \mathrm{~s}^{-1}$ per ${ }^{\circ} \mathrm{C}$, (b) temperature compensated data, applying a temperature coefficient of $0.91 \mathrm{~m} \mathrm{~s}^{-1} \mathrm{per}^{\circ} \mathrm{C}$, (c) temperature compensated data, estimated from the malic and lactic acid concentrations.

\subsection{Temperature compensation in real wine samples}

4.3.1. Introduction. The temperature compensation coefficients are obtained using two different methods, as described in section 4.2. These coefficients are applied to compensate for the temperature effect in the two real wine samples under study. This section discusses the results obtained.

4.3.2. Palencia wine sample. The first wine sample studied corresponds to a MLF elaborated in a pilot plant in Palencia. If the temperature coefficients of compensation obtained in section 4.2.2 are applied, it is possible to estimate the ultrasonic velocity of propagation after the removal of the temperature contribution (figures 11(a) and (b)). Also, malic and lactic acid concentrations are provided for this wine sample, obtained by enzymatic methods (see section 2.1.3). In a previous paper (Amer et al 2015), the authors described an empirical equation that correlates the ultrasonic velocity of propagation with the malic and lactic acid concentrations. Accordingly, it is possible to estimate the ultrasonic velocity of propagation from the malic and acid concentration. The results obtained are also shown in figure 11(c).

The experimentally obtained results (plots (a) and (b)) show that the ultrasonic velocity variation initially increases, followed by a decrease, and ends up increasing again until a new stable value is reached, which is higher than the initial value. It is also observed that the ultrasonic velocity variation calculated after applying a temperature compensation coefficient is significantly lower than the measured values from figure 6, and close to the expected theoretical results (plot (c)).

In figure 11, the differences between plots (a) and (b) are basically due to the different coefficients applied to compensate for temperature variations, obtained by the two different methods previously described (section 4.2). Because the values of both coefficients are similar, the differences between plots (a) and (b) are small and less than $0.1 \mathrm{~m} \mathrm{~s}^{-1}$ (which is the uncertainty of the measurement method of ultrasonic velocity). 


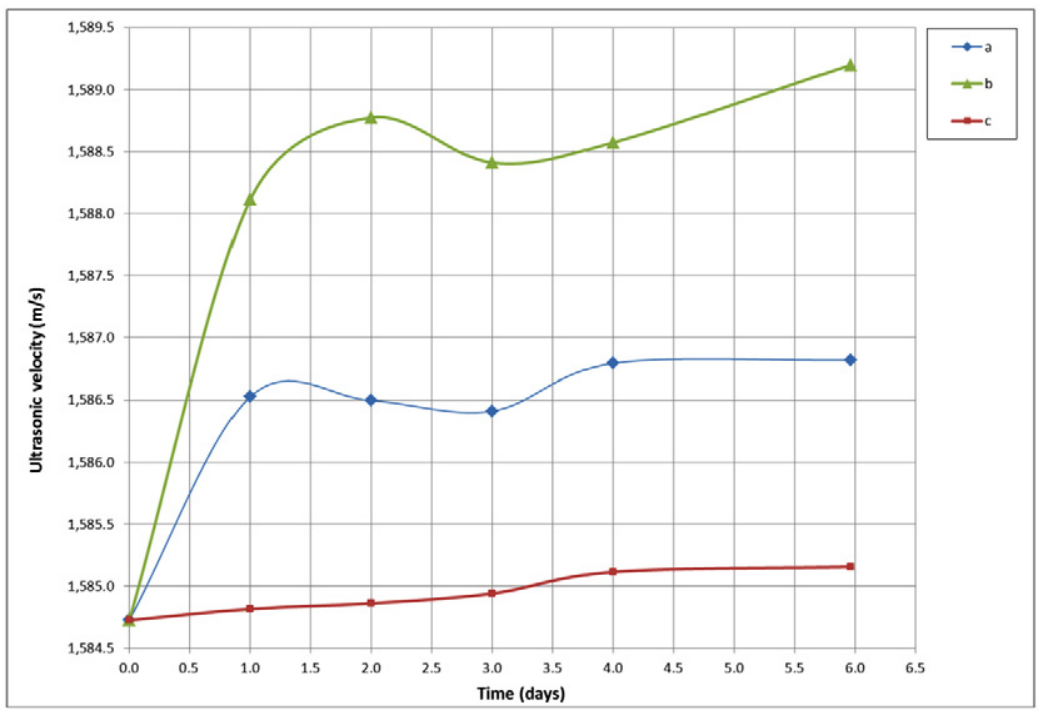

Figure 12. Ultrasonic velocity of propagation in the Penedès wine sample, among time. (a) Temperature compensated data, applying a temperature coefficient of $0.52 \mathrm{~m} \mathrm{~s}^{-1}$ per $^{\circ} \mathrm{C}$, (b) temperature compensated data, applying a temperature coefficient of $0.96 \mathrm{~m} \mathrm{~s}^{-1}$ per ${ }^{\circ} \mathrm{C}$, (c) temperature compensated data, estimated from the malic and lactic acid concentrations.

Moreover, the theoretical results (plot (c)), estimated from the malic and lactic acid concentrations, show that the ultrasonic velocity variation along the MLF is quite small (only $0.6 \mathrm{~m} \mathrm{~s}^{-1}$ approximately). If this value is compared with the temperature coefficient of compensation (which is about $0.9 \mathrm{~m} \mathrm{~s}^{-1}$ per degree Celsius), a temperature change of only one degree Celsius can cause a more significant change in the ultrasonic velocity than the change in the ultrasonic velocity due to the malic and lactic acid variations along the entire MLF. Once again, this highlights that changes in temperature can seriously mask the variations due to changes in malic and lactic acid concentration and make it more difficult to monitor the MLF (as happens in fact).

Also, from figure 11, the estimated values (plot (c)) may be compared with the measured ones once the temperature effect was compensated (plots (a) and (b)). It is observed that all curves vary similarly after a time of $100 \mathrm{~h}$, but not before. These differences between the estimated and measured curves were justified in a previous paper (Amer et al 2015), and they were related to the changes that take place during the bacterial growth phase (i) (see section 2.1.2). After the bacterial growth phase (i), stationary phase I (ii) takes place, when malic acid is turned into lactic acid and both curves (estimated and measured) are similar in this period of time. When this phase finishes, no more malic acid is turned into lactic acid, and the ultrasonic velocity should remain constant. This may be used to detect the end point of the MLF.

4.3.3. Penedès wine sample. The second wine sample corresponds to a real MFL produced in Penedès. Figure 12 shows the ultrasonic velocity of propagation after the temperature effect is compensated (plots (a) and (b)). Also, and similar to Palencia, from the malic and lactic acid concentrations obtained by enzymatic methods (one sample every $24 \mathrm{~h}$ ), the theoretical ultrasonic velocity was estimated, as depicted in figure 12(c).
In figures 12(a) and (b), it is observed that the ultrasonic velocity initially increases quickly (during the first day, and more pronounced than in Palencia) until it reaches a maximum, then decreases to a minimum, and finally increases again. These signals have similar profiles to the Palencia ones, and differences between curves $\mathrm{a}$ and $\mathrm{b}$ are due to the coefficients applied, $0.52 \mathrm{~m} \mathrm{~s}^{-1}$ per ${ }^{\circ} \mathrm{C}$ and $0.96 \mathrm{~m} \mathrm{~s}^{-1}$ per ${ }^{\circ} \mathrm{C}$, respectively. Because the values of both coefficients are quite different, differences between curves a and $\mathrm{b}$ are significant.

As it happens in Palencia, the theoretical ultrasonic velocity variation along the MLF, estimated from the malic and lactic acid concentrations (figure 12(c)), is quite small (only $0.6 \mathrm{~m} \mathrm{~s}^{-1}$ approximately). This value is similar to the temperature coefficient of compensation, obtained from correlating the ultrasonic velocity and temperature in real wine samples $\left(0.52 \mathrm{~m} \mathrm{~s}^{-1}\right.$ per degree Celsius). So, a change of only one degree Celsius causes the same ultrasonic velocity variation than the one caused by the acid concentrations during the MLF. Because temperature variations between night and day are about $8{ }^{\circ} \mathrm{C}$ in one day, velocity changes due to temperature variations during one day are about 8 times the ultrasonic velocity changes due to the malic and lactic acid concentration changes throughout the entire MLF. This makes it more difficult to monitor the MLF.

So, from figure 12 , the estimated values may be compared with the measured ones once the temperature effect was compensated for. Significant differences between plots are observed. Again, disagreements between the estimated and measured ultrasonic velocities at the beginning of the MLF might be due to factors related to the bacterial growth phase, and not to changes in the lactic and malic acid concentrations (as previously commented). When stationary phase I (ii) takes place, malic acid is turned into lactic acid, and plots a and $\mathrm{c}$ are similar during this period of time. When this phase finishes, no more malic acid is turned into lactic acid, and the ultrasonic 
velocity remains constant. Again, this fact may be used to detect the end point of the MLF.

Moreover, if figures 12(a) and (b) are compared, another significant difference is appreciated. In contrast to plot (a), a final asymptote in plot (b) is not appreciated, which might indicate the end of the fermentation. So, from plot (b) it is not possible to detect when the fermentation is finished.

As a conclusion, in the Penedès wine sample and despite the significant limitations observed, the temperature coefficient of compensation obtained from correlating the ultrasonic velocity and temperature $\left(0.52 \mathrm{~m} \mathrm{~s}^{-1}\right.$ per degree Celsius) gives better results than the value obtained from the alcoholic degree and applying equation (5) $\left(0.96 \mathrm{~m} \mathrm{~s}^{-1}\right.$ per degree Celsius).

\section{Conclusions}

Temperature is the most significant factor that affects the ultrasonic velocity variation during the MLF process. Therefore, measurements of the ultrasonic velocity variation due to changes in malic and lactic acid concentrations are masked by temperature variations. Two temperature compensation methods are applied to the measured ultrasonic velocity values from two different malolactic fermentation processes. The temperature compensation coefficient obtained by 'on-line' correlating the ultrasonic velocity and the temperature during the MLF process provides much better results and demonstrates the applicability and validity of this technique.

Although these results are quite promising, it must be taken into account that this work is a preliminary study. The results obtained during the initial phase of the MLF do not fit with the theoretical ones. Therefore, in order to develop a commercial prototype further research is required.

\section{Acknowledgments}

This work is financially supported by the Spanish Ministerio de Economía y Competitividad Project with reference

\section{References}

Amer M A, Novoa-Díaz D, Puig-Pujol A, Capdevila J, Chávez J A Turó A, García-Hernández M J and Salazar J 2015 Ultrasonic velocity of wáter-ethanol-malic acid-lactic acid mixtures during the malolactic fermentation process J. Food Eng. 149 61-9
García-Álvarez J, Novoa-Díaz D F, Bertran E, Chávez J A, Puig-Pujol A, Turó A, Mínguez S, García-Hernández M J and Salazar J 2011 Ultrasonic study of red wine properties: preliminary measurements 34 th World Congress of Vine and Wine. Proc. 34th World Congress of Vine and Wine: the Wine Construction (Porto, Portugal, 20-27th June 2011) pp 1-7

Gross V A D and Mader C W 1972 Speed of sound in pure water J. Acoust. Soc. Am. 52 1442-6

Krieger S 2006 Determining when to add malolactic bacteria Malolactic Fermentation in Wine-Understanding the Science and the Practice (Montréal: Lallemand) ed R Morenzoni pp 12.1-10

Lerm E, Engelbrecht L and du Toit M 2010 Malolactic fermentation: the ABC's of MLF South Afr. J. Enol. Viticulture 31 186-212

Lonvaud-Funel A 2004 Bacterias lácticas y fermentación maloláctica El vino y su Industria 5 42-50

Liu S Q 2002 A review: malolactic fermentation in wine-beyond deacidification J. Appl. Microbiol. 92 589-601

Maicas S, Gil J-V, Pardo I and Ferrer S 1999 Improvement of volatile composition of wines by controlled addition of malolactic bacteria Food Res. Int. 32 491-6

McClements D J 1997 Ultrasonic characterization of foods and drinks: principles, methods and applications Crit. Rev. Food Sci. Nutrition 37 1-46

Novoa-Díaz D, García-Álvarez J, Chávez J A, Turó A, GarcíaHernández M and Salazar J 2012 Comparison of methods for measuring ultrasonic velocity variations during ageing or fermentation of food materials IET Sci., Meas. Technol. $6205-12$

Novoa-Díaz D, Rodríguez-Nogales J M, Fernández-Fernández E, Vila-Crespo J, García-Álvarez J, Amer M A, Chávez J A, Turó A, García-Hernández M J and Salazar J 2014a Ultrasonic monitoring of malolactic fermentation in red wines Ultrasonics 54 1575-80

Novoa-Díaz D 2014b Desarrollo de un sistema de medida ultrasónico para monitorizar el proceso de fermentación maloláctica del vino tinto en un ambiente industrial $P h D$ Dissertation Universitat Politècnica de Catalunya, Spain

OIV 2012 Recueil des Méthodes Internationales d'Analyse des Vins et des mouts Office International de la Vigne et du Vin, Paris, France

Resa P, Bolumar T, Elvira L, Pérez G and Montero de Espinosa F 2007 Monitoring of lactic acid fermentation in culture broth using ultrasonic velocity J. Food Eng. 78 1083-91

Salazar J, Chávez J A, Turó A and García-Hernández M J 2009 Ultrasound effect on food processing Novel Food Processing: Effects on Rheological and Functional Properties ed J Ahmed et al (Boca Raton, FL: CRC Press)

Vila-Crespo J, Rodriguez-Nogales J M, Fernández-Fernández E and Hernanz-Moral M C 2010 Strategies for the enhancement of malolactic fermentation in the new climate conditions Curr. Res. Technol. Educ. Top. Appl. Microbiol. Mater. Biotechnol. 2920-9

Wibowo D, Eschenbruch R, Davis C R, Fleet G H and Lee T H 1985 Occurrence and growth of lactic acid bacteria in wine: a review Am. J. Enol. Viticulture 36 302-13 